\title{
Intracranial Germ Cells Tumour: a single institution experience in Argentina
}

\section{Lorena Viviana Baroni}

Paediatric Hospital Dr Juan Garrahan: Hospital de Pediatria Prof Dr Juan P Garrahan

Agustina Oller ( $\sim$ agustinaoller@gmail.com )

Paediatric Hospital Dr Juan Garrahan: Hospital de Pediatria Prof Dr Juan P Garrahan https://orcid.org/0000-0001-8183-3167

\section{Candela Soledad Freytes}

Paediatric Hospital Dr Juan Garrahan: Hospital de Pediatria Prof Dr Juan P Garrahan

\section{Claudia Vanesa Sampor}

Paediatric Hospital Dr Juan Garrahan: Hospital de Pediatria Prof Dr Juan P Garrahan Nicolas Ponce Fernandez

Paediatric Hospital Dr Juan Garrahan: Hospital de Pediatria Prof Dr Juan P Garrahan Natalia Pinto

Paediatric Hospital Dr Juan Garrahan: Hospital de Pediatria Prof Dr Juan P Garrahan

\section{Carlos Rugilo}

Paediatric Hospital Dr Juan Garrahan: Hospital de Pediatria Prof Dr Juan P Garrahan

\section{Fabiana Lubieniecki}

Paediatric Hospital Dr Juan Garrahan: Hospital de Pediatria Prof Dr Juan P Garrahan

\section{Pedro Zubizarreta}

Paediatric Hospital Dr Juan Garrahan: Hospital de Pediatria Prof Dr Juan P Garrahan

\section{Daniel Horacio Alderete}

Paediatric Hospital Dr Juan Garrahan: Hospital de Pediatria Prof Dr Juan P Garrahan

\section{Clinical Study}

Keywords: Germ-cell tumor, germinoma, non germinomatous, brain tumor, reduced radiation field

Posted Date: February 3rd, 2021

DOl: https://doi.org/10.21203/rs.3.rs-157912/v1

License: (c) (i) This work is licensed under a Creative Commons Attribution 4.0 International License. Read Full License 
Version of Record: A version of this preprint was published at Journal of Neuro-Oncology on March 3rd, 2021. See the published version at https://doi.org/10.1007/s11060-021-03709-7. 


\section{Intracranial Germ Cells Tumour: a single institution experience in Argentina}

Lorena V. Baroni ${ }^{1 \#}$, Agustina Oller ${ }^{1 \#}$,Candela S. Freytes ${ }^{1}$, Claudia V. Sampor ${ }^{1}$,Natalia Pinto $^{2}$,Nicolas Ponce Fernandez ${ }^{1}$ Carlos Rugilo ${ }^{3}$, Fabiana Lubieniecki ${ }^{4}$, Pedro Zubizarreta ${ }^{1}$, Daniel Alderete ${ }^{1}$

1) Service of Hematology/Oncology, Hospital JP Garrahan, Buenos Aires, Argentina (LB, $\mathrm{AO}, \mathrm{CS}, \mathrm{CF}, \mathrm{DA}, \mathrm{NP}, \mathrm{PZ})$

2) Service of Radiotherapy, Hospital JP Garrahan, Buenos Aires, Argentina (NP)

3) Service of Diagnostic Imaging, Hospital JP Garrahan, Buenos Aires, Argentina (CR)

4) Service of Pathology, Hospital JP Garrahan, Buenos Aires, Argentina (FL)

\section{\# denotes co-authorship}

To Whom Correspondence may be addressed

Dr. Daniel Alderete

1881 Combate de los Pozos Street

Buenos Aires, Argentine

Danalder09@gmail.com

Dr. Lorena Baroni

ORCID ID: 0000-0002-1599-7080

1881 Combate de los Pozos Street 
Buenos Aires, Argentine

lorelein@msn.com

Dr. Agustina Oller

ORCID ID:0000-0001-8183-3167

1881 Combate de los Pozos Street

Buenos Aires, Argentine

agustinaoller@gmail.com

Declarations

Funding: No funding

Conflict of interest: The authors declare no conflicts of interest.

Availability of data and materials: Data available within the article or its supplementary materials

Code availability: Not applicable

Authorship: Conceptualization: LB, AO, DA; Methodology: LB, AO, DA; Investigation, LB, DA, AO; Data Curation: LB, AO, NP, CS, CF, FL, CR; Formal Analysis: LB, AO; Writing Review \& Editing: LB, AO; Resources: LB, AO, DA, CS, CF, FL, CR., Project Administration: LB, DA; Supervision: PZ, DA

Total manuscript word count: $3147 \quad$ Abstract: 306 
Tables: 4

Figure: 1

Supplementary table: 1

\begin{abstract}
Background: Intracranial germ cell tumor (iGCT) represents a rare and heterogeneous group, with variable incidence and diverse treatment strategies. Although multiagent chemotherapy with reduced radiotherapy strategy has been applied by several cooperative groups in North America and Western Europe, there is a paucity of data to understand if this combined regimen issuitable in low-middle income countries (LMIC).
\end{abstract}

Methods: We evaluate the outcome in a cohort of iGCT treated by SIOP-CNS-GCT-96 strategy at Hospital J.P Garrahan in Argentinaover the last 20 years. Radiation field and dose included focal radiotherapy (FRT) before 2009 or focal radiotherapy plus whole ventricular radiotherapy (WVRT) after 2009 for localized germinoma and FRT or FRT plus WVRT or CSI for non germinomatous germ cell tumors (NGGCT)

Results: Sixty iGCT were identified; 39 germinoma and 21 NGGCT. Median follow-up was 6.57 years (range 0.13-20.5). Five-year PFS and OS were $83.5 \%$ (95\% CI [165.53 - 223.2]) and $88.7 \%$ (95\% CI [169.84 - 223.2]) for the germinoma group, while for the NGGCT group were 75\% (95\% CI [133.27 $219.96])$ and $64.2 \%$ (95\% CI [107.38 - 201.81]) respectively. The localized germinoma group showed poor results between 2000-2009 with 5-year PFS and OS of 69\% and 75\% respectively, and an excellent outcome between 2010-2019 with a 5-years PFS and OS of $92.8 \%$ and $100 \%$. A univariable analysis identified this difference in survival as related to the field of radiotherapy, specifically whole ventricular radiotherapy. FRT increased the risk of recurrence in localized germinoma, involving not only 
ventricular relapses; but spinal cord and disseminated disease as well. There were no relapses of localized NGGCT after FRT and FRT plus WVRT.

Conclusion: Herein we demonstrate that intensive chemotherapy followed by FRT plus WVRT for germinoma is a feasible and effective strategy, warranting further study in the developing world.

Keywords: Germ-cell tumor, germinoma, non germinomatous, brain tumor, reduced radiation field 


\section{Introduction}

Intracranial germ cell tumor (iGCT) represents a rare and heterogeneous group, with variable incidence and diverse treatment strategies across the continents [1-2]. Histologically, iGCT are classified into 7 types by the 2016 World Health Organization guidelines: germinomas, embryonal carcinomas, yolk sac tumors, choriocarcinomas, teratomas, teratomas with malignant transformation, and mixed germ cell tumors [3]. However, the initial surgical management is based on the levels of specific tumor markers in serum and cerebrospinal fluid concentration (CSF). Historically, alpha-fetoprotein (AFP) and human chorionic gonadotropin-beta (hCG $\beta$ ) classified iGCT in Europe and the Americas, in two groups: germinoma and non-germinomatous germ-cell tumours (NGGCT). This classification system reflects the excellent overall survival for the first group, and the inferior survival for the second one. Most recently, concentration of AFP more than 1000kU/l identified a high risk NGGCT subgroup with a poor outcome that may be benefited from intensification of therapy[4-5].

Although some international consensus in the management of iGCT has been achieved, several challenges persist[6-7]. Currently, the role of surgery at diagnosis is limited to surgical intervention for obstructive hydrocephalus or acute visual deterioration and/or biopsy in marker-negative cases. Radiotherapy is almost ubiquitously prescribed across all cases of germinoma and NGGCTin order to achieve satisfactory survival[8]. Despite efforts to substitute radiation with chemotherapy only, the high relapse rates of up to $50 \%$ and inferior outcome, suggested this is an unacceptable treatment approach for iGCT [9-10-11]. Being highly radiation sensitive, all patients with germinomas were historically treated with craniospinal irradiation (CSI) alone, which yielded a cure rate of more than $90 \%$ [12]. However, this approach results in severe long-term toxicities, such as neurocognitive impairment and endocrinopathies, which has led to studies on reduced radiotherapy dose and field to minimise the risk of late toxicities while maintaining excellent prognosis [13-14]. On the other hand, historical series of NGGCT treated by radiotherapy alone reported $20-40 \%$ overall survival suggesting that a multimodal approach was required[15]. Chemotherapy has been used for the treatment of both germinoma and NGGCT, helping to 
reduce radiotherapy fields or doses, or both, during germinoma treatment while improving outcomes substantially for NGGCT.

The strategies employed by the various cooperative groups varied widely.Specifically, the European strategyhascompared CSI to a chemotherapy and focal radiation approach in localized germinoma yielding a 5-year progression free survival of 97 and $88 \%$ respectively, North America (ACNS 0232/1123) has employed chemotherapy followed by whole ventricular radiotherapy (WVRT) and focal boost and the Japanese group a chemotherapy strategy consisting of carboplatin and etoposide followed by ventricular radiation; both strategies yielding a comparable survival. When analysing our cohort we found that previous to 2009, our treatment strategy was similar to European approach and after 2009 treatment was based on North American approach. On the other hand, the European strategy for NGGCT consists of a cisplatin-based chemotherapy, followed by surgical resection if residual disease is present and then focal radiotherapy, while North America (ACNS0122) has employed a carboplatin-based chemotherapy followed by craniospinal radiotherapy and focal boost, and the Japanese group employs concomitant chemoradiotherapy with ICE (Ifosfamide-Carboplatin-Etoposide) and radiotherapy to the tumour bed followed by CSI and ICE chemotherapy [8-16-17].

The European Société Internationale d'Oncologie Pédiatrique (SIOP) showed that chemotherapy followed by local field radiotherapy presented a good initial response in germinoma tumours, but this treatment was insufficient to control subependymal growth in the ventricular area suggesting the need to enlarge the radiation field to include the ventricles. Moreover, they did not identify the presence of residual lesions at the end of treatment as an adverse prognostic factor in germinoma showing an excellent survival for the total cohort [18]. On the other hand, SIOP-CNS-GCT-96 strategy showed that combination chemotherapy and radiotherapy for all intracranial NGGCT patients, with risk-adapted radiotherapy tailored according to initial dissemination (focal for those with localized disease and craniospinal plus focal boost for metastatic cases), is effective in producing long term durable treatment responses with a five-year progression free survival of $72 \%$ and $68 \%$ for localized and metastatic disease, respectively. In contrast to 
the germinoma experience, they identified that survival for patients with intracranial malignant NGGCTs who had end-of-treatment residual disease (including after second-look surgery) was significantly worse [19].

Most reports on pediatric central nervous system tumors from LMIC describe the epidemiology of these tumors and rarely describe in detail treatment modalities and outcomes. Due to the limitation of health care resources, protocols in LMIC should not only consider survival, but also feasibility. The treatment of malignant childhood brain tumours remains a challenge in LMIC as it requires a multidisciplinary team that may not available worldwide, such as access to chemotherapy, surgical expertise, supportive care and radiation facilities.Although the SIOP-CNS-GCT-96 strategy is followed by many countries across the world, the viability and survival outcome in theLMICsetting is not well described.Despite small series [20-22], there is a paucity of data to understand if this combined regimen would be considered a possible and practicable strategy outside the developed world. Here, we evaluate the outcome of a cohort of iGCT treated using the SIOP-CNS-GCT-96 approach prior to 2009 followed by the adoption of the North American approach of whole ventricular radiotherapy after 2009 at Hospital JP Garrahan in Buenos Aires.

\section{Methods}

\section{Patients and treatment}

This retrospective study was performed in accordance with the approval of the Hospital JP Garrahan Research Ethics Board. The treatment strategy was used as a standard of care from 2000-2020 in Hospital JP Garrahan. All patients had AFP and hCG $\beta$ level in serum and/or CSF as initial management. "Secreting” or malignant NGGCT was defined as per SIOP regimen with serum or CSF elevation of AFP more than $25 \mathrm{ng} / \mathrm{mL}$ and/or serum CSF elevation of $\mathrm{hCG} \beta$ more than 50UI/ml and /or biopsy demonstrating nongerminomatous elements (yolk sac tumor, embryonal carcinoma, choriocarcinoma, teratoma with malignant transformation, and mixed malignant germ cell tumour). Allgerminoma were 
diagnosed based on biopsy performed in the context of levels of the tumour markers AFP and hCG $\beta$ within the defined limits stated above. Patients were staged with craniospinal imaging and lumbar CSF cytology at baseline unless clinically contra-indicated. Positive CSF-cytologyand/or positive imaging was defined as metastatic disease. Synchronous bifocal intracranial tumors (defined as masses in the pineal and suprasellar region only by magnetic resonance imaging) were considered non-metastatic disease and were treated in the same way as unifocal tumours. Although a European consensus has been reached that a biopsy is not mandatory in bifocal tumours with typical appearance, all of them had histological verification in our cohort.Except for 1 localized germinoma who received CSI alone, all patients received chemotherapy followed by radiotherapy. Neoadjuvant chemotherapy was based on SIOP-CNS-GCT 96 (2 courses of carboplatin/etoposide alternating with Ifosfamide/Etoposidefor germinoma and 4 courses of Cisplatin/Etoposide/Ifosfamide for NGGCT) in 55 patients while 4 patients received 4 courses of Carboplatin/Etoposide (avoiding Ifosfamide) for better diabetes insipidusmanagement. Radiation field and dose changed over the years and as per radiotherapistconsideration, FRT was defined as radiation delivered to the tumor bed at the time of diagnosis, including any residual tumor present at the time of treatment planning. The WVRT encompassed the lateral, third, and fourth ventricles, including the suprasellar and pineal cisterns. In reporting radiotherapy fractionations, equivalent dose in 1.8-Gy (LQ model) was used with an alpha/beta ratio of 10 [23-24]. Radiation fields and median radiotherapy doses are shown in supplementary Table 1.

Tumor markers were performed before each cycle of chemotherapy, after radiation therapy and every 4 months after treatment. Neuroimaging was performed after two initial cycles and after finishing chemotherapy, 4-6 weeks after the end of radiotherapy, every 4-6 months for 3 years and yearly after that.

\section{Statistical analysis}

Descriptive statistics were used to report baseline patient, tumorand treatment characteristics. Progression free survival (PFS) was defined as the time between diagnosis and either relapse or last follow up contact for patients who did not experience a recurrence. Overall survival (OS) was defined as the time between 
diagnosis and death from any cause or last follow up contact for patients who were alive. PFS and OS were calculated using the Kaplan-Meier method and reported at 5 years. Survival curves were compared using the log-rank test. All statistical analyses were performed in Graph Pad Prism 8.For the analysis, FRT and FRT plus WVRT were considered reduced-volume radiotherapy.

\section{Results}

\section{Demographics of the entire cohort}

Sixty consecutive patients with iGCT at our institution were treated between September 2000-October 2019 (28 between 2000-2009; 32 between 2010-2019). Median time of follow-up was 6.57 years (range 0.13-20.5). Demographic and disease characteristics of the entire cohort (39 germinomas and 21 NGGCT) are summarized in Table 1. Initial surgery was conducted in 49 patients, 39 were biopsies (32=endoscopic, $3=$ Stereotactic, open surgery=4), 8 partial resections and 2 gross total resections.No patient died due to surgical complications and the median time between diagnosis and the first course of chemotherapy was 49 days (range 9-79). Residual tumor was described in 19 patients after completing chemotherapy (germinoma=7; NGGCT=12) and 9 after radiotherapy (germinoma=3; NGGCT=6); two of them had a second look surgery as part of the treatment strategy (germinoma=1, NGGCT=1).

\section{Germinoma and NGGCT specific outcome}

Five-year PFS and OS for the total cohort were $80 \%$ (95\% CI [164.52 - 213.44]) and 80.5\% (95\% CI [158.43 - 207.78]) respectively. Five-year PFS and OS were 83.5\% (95\% CI [165.53 - 223.2]) and 88.7\% (95\% CI [169.84 - 223.2]) for the germinoma group(Figure A), being 78\% (95\% CI [155.18 - 207]) and $88 \%$ (95\% CI [156.18 - 210-03]) for the localized subgroup(excluding the only patient who received CSI without chemotherapy) (Figure C) and 100\% for the metastatic subgroup. Localized germinoma group showed poor results between 2000-2009 but excellent outcome between 2010-2019, having a 5-year PFS 
of $69 \%$ in the first cohort and $92.8 \%$ in the second cohort (Figure 1D; p=0.17 HR $3.395 \% \mathrm{CI}[0.6786$ 13.59]) and a 5-year OS of $75 \%$ in the first cohort and $100 \%$ in the second cohort (Figure 1E; $\mathrm{p}=0.10 \mathrm{HR}$ 4.7 95\% CI [0.734 - 31.06]). Five-year PFS for localized germinoma who received FRT was 63\% while for the subgroup who received FRT plus WVRT was 94\% (Figure 1F; p=0.07 HR 4.8 95\% CI [0.96 24.31]).

Five-year PFS and OS were 75\% (95\% CI [133.27 - 219.96]) and 64.2\% (95\% CI [107.38 - 201.81]) NGGCT group, being $61 \%$ and $62.8 \%$ for the localized NGGCT subgroup and $100 \%$ for the metastatic subgroup. Localized NGGCT group showed similar results between 2000-2009 and 2010-2019, having a 5-year PFS of $60 \%$ in the first cohort and $67.5 \%$ in the second cohort ( $\mathrm{p}=0.9$ HR $1.1795 \%$ CI $[0.21$ 6.54]). There were no relapses of localized NGGCT after reduced-volume radiotherapy treatment (FRT and FRT plus WVRT).

\section{Relapses}

Our relapsed germinoma cohort represents a relatively small and heterogeneous group, in terms of type of relapse (marker-negative vs. marker-positive) and relapse treatments delivered (standard dose chemotherapy vs HD-SCR; re-irradiation vs. none). During the first 24 months after diagnosis, 5 patients with localized Germinoma (bifocal=2, sellar/suprasellar=2, pineal=1) experienced relapse at a median time of 20.9 months. Late relapse (by definition at $\geq 2$ years after successful treatment) was observed in two localized germinoma (bifocal=1, sellar/suprasellar=1) at 108 and 145 months. No biopsy was performed at relapse. Three relapses had high tumor markers levels at recurrence, but none of them at initial diagnosis (Table 1 and Table 2). While five of the seven relapses had received FRT as initial management, 2 had received FRT plus WVRT. Two patients had isolated intraventricular relapse; both had received FRT upfront.Three patients had spinal lesions at recurrence (isolated=1, in combination with others supratentorial lesions $=2$ ); all of them had received reduced-volume radiation at diagnosis $(\mathrm{FRT}=1$, FRT plus WVRT=2) (Table 2). The majority of relapsed germinomas treated with curative intention received re-induction chemotherapy with a platinum-based (cisplatin - carboplatin) strategy followed by 
CSI as $1^{\text {st }}$ line salvage therapy $(\mathrm{N}=5)$. One marker positive relapsed germinoma received four cycles of Gemcitabine-Paclitaxel-Oxaliplatin (GEMPOX) followed by high dose chemotherapy and stem cell rescue as a second line salvage treatment with tumor remission for 30 months after second recurrence. Four of the germinoma's relapse patients died, 3 due to tumor progression and 1 associated with a second tumor (Table 2).

Four patients with localized NGGCT (pineal=2, thalamus=1, intraventricular=1) progressed during multiagent chemotherapy treatment (SIOP strategy) and 1 (pineal) immediately after end of treatment. No late relapses were observed in this group. Three patients had high risk serum AFP levels (>1000 ng/ mL) at diagnosis and 1 had evidence of residual disease after chemotherapy. Two patients with NGGCT recurrence received GEMPOX regimen and showed an initial response but a rapid progression after 3-4 cycles. Although different approaches had been used, all NGGCT patients died after relapse showing the poor prognosis of this particular group (Table 3).

\section{Causes of death}

Two patients (germinoma=1, NGGCT=1) died after the first cycle of chemotherapy (carboplatinEtoposide) due to a systemic infection disease at 1.4 and 1.7 months from diagnosis. One patient with localized NGGCT with panhypopituitarism since diagnosis and poor treatment adherence, died regarding a diabetes insipidus disorder at 21 months without tumor recurrence. Three patients with germinoma (localized=2, metastatic=1) developed a second tumor; 1 acute myeloid leukemia M5 and 2 posterior fossa tumors (1 medulloblastoma and 1 malignant tumor no specify). These last two patients received CSI; 1 as part of the metastatic treatment regimen, and 1 as part of the first salvage strategy. No family history of cancer predisposition syndrome was found (Table 4).

\section{Discussion}

Herein we report the outcome of localized germinoma and NGGCT treated with multi-agent chemotherapy (carboplatin-based chemotherapy and cisplatin-based chemotherapy respectively), 
followed by reduced-volume radiation during 20 years in one center in Argentina. In addition, treatment results involving differences in radiation field prior to 2009 and after that date are described. We show that whole ventricular radiotherapy is required to achieve high survival rates in germinoma, and focal radiotherapy alone is insufficient for this group. Our study also confirms the feasibility of treating a subset of NGGCT with craniospinal irradiation sparing protocols in the developing world, suggesting this is as a global option to balance survival with acceptable quality of life.

In order to preserve quality of survival highly curable disease like localized CNS germinoma, we employed a chemotherapy strategy followed by reduced-volume radiotherapy, reserving craniospinal radiation for cases with metastatic dissemination [18]. This strategy was previously described by several cooperative groups in Europe and Japan with excellent survival [5]. While the cohort between 2000-2009 showed an inferior PFS and OS outcome, the cohort between 2010-2019 demonstrated a similar favourable survival data to SIOP experience. Although chemotherapy was based on carboplatin in both cohorts, radiotherapy fields differed. When radiation fields were compared, we found that focal radiation alone increased the risk of recurrence, involving not only ventricle relapses; but spinal cord and disseminated disease as well. In our cohort WRTV following chemotherapy is likely to be a safe treatment to control subclinical disease, consistent with previous reports where a local field radiotherapy has shown to be insufficient to control subependymal growth in the ventricular area and increased the risk of ventricular relapse [27-29].

In our experience,NGGCT recurred on chemotherapy or immediately after the end of treatment. All recurrences had been locally and/or with high tumor markers levels. In contrast with ACNS0123 trial results [30], where the preponderance of distant relapse had been concerning; we did not find any distant relapse (including the subgroup who received FRT or FRT plus WVRT upfront), showingthat craniospinal radiotherapy might be avoided without increased relapses outside the radiotherapy field. Consistent with previous SIOP-CNS-GCT-96 trial results [19], serum/ CSF AFP level $>1000 \mathrm{ng} / \mathrm{mL}$ at diagnosis was associated with a negative prognostic impact in our cohort, confirming this as a marker of poor prognosis(p=0.009 HR $2295 \%$ CI [2.13-228]). 
Our study has the classic limitations of a retrospective study, where there are missing observations, including isolated cases where staging was incomplete. Specifically, there were two relapsed germinoma's where CSF tumor markers at diagnosis were not performed due to concerns around lumbar CSF examinations, and as such we cannot completely dismiss the possibility that these were misdiagnosed NGGCT. Nevertheless, our overall trend that the radiation field is a predictor of outcome is highly significant, and our study confirms the feasibility of a craniospinal irradiation avoiding approach for NGGCT. However, as a single centre consecutive cohort, our follow up was uniform, and as such, our study further confirms that the SIOP-CNS-GCT 96 approach results in excellent outcome in the developing world. A second limitation is a lack of uniform neurocognitive outcomes in our cohort, which is a crucial determinant moving forward to justify de-escalations of therapy - and more importantly a lack of uniform neurocognitive outcomes collected by the cooperative group trials.

Another important consideration are the three late deaths in the germinoma group after a second malignancy without evidence of germ cell tumor recurrence. Secondary leukemia after treatment with a conventional dose of etoposide has been well described, but the low incidence in patients who receive lower doses $\left(\leq 2 \mathrm{gr} / \mathrm{m}^{2}\right)$ does not alter the risk-to-benefit ratio of etoposide-based chemotherapy in germ cell cancer [31]. Topoisomerase II inhibitor-related secondary leukemias are generally diagnosed 2 to 3 years following treatment, most commonly exhibit M4 or M5 phenotype and have a poor prognosis [32]. On the other hand,both with solid second neoplasm received radiotherapy in that area; 1 as part of the primary treatment disease and 1 as part of the salvage treatment. Despite no obvious family history of cancer and the paucity of literature on germline mutations and germ cell tumors treated with radiation, we cannot exclude the possibility that these second tumors could have been related an underlying cancer predisposition.

Managing iGCT in LMIC poses a unique set of challenges including access to tumor markers screening, stage at presentation, adequacy of management and availability of therapeutic interventions. Optimal chemotherapy therapy was administered in all iGCT patients without delay. While proton therapy is not available in Argentina, intensity modulated radiotherapy and 3D-conformal radiotherapy 
are the two techniques most frequently used since 2015 at our institution. On the other hand, abandonment treatment rates seem to be higher in LMIC however, all iGCT's patients were able to complete therapy without delays [33]. Implementing a patient-tracking system, monitoring missed appointments, and providing resources to help families during treatment prevented unnecessary treatment interruption.

Despite the limitations of analysing historical series, this study fills a gap in the literature on iGCT outcome and treatment patterns at a single institution in the developing world. Our experience suggests that adjuvant chemotherapy followed by focal radiotherapy results in inferior survival compared to whole ventricular radiotherapy, and confirms the feasibility of this approach in a low-middle income setting. Future collaborative studies in low-middle income countries might contribute to define better standards of care in our setting and help improve quality of life for this group of highly curable central nervous system malignancies. 


\section{References}

1. Osorio DS, Allen JC (2015) Management of CNS germinoma. CNS oncology4(4):273-9.

2. Fetcko K, Dey M (2018) Primary Central Nervous System Germ Cell Tumors: A Review and Update. Med Res Arch 6(3):1719.

3. Kong Z, Wang Y, Dai C, Yao Y, Ma W, Wang Y (2018) Central Nervous System Germ Cell Tumors: A Review of the Literature. Journal of child neurology33(9):610-620.

4. Göbel U, Calaminus G, Schneider DT, Schmidt P, Haas RJ (2002) Management of germ cell tumors in children: approaches to cure. Onkologie 25(1):14-22.

5. Bowzyk Al-Naeeb A, Murray M, Horan G, et al (2018) Current management of intracranial germ cell tumours. Clin Oncol (R Coll Radiol) 30(4):204- 214.

6. Abu Arja MH, Bouffet E, Finlay JL, AbdelBaki MS (2019) Critical review of the management of primary central nervous nongerminomatous germ cell tumors. Pediatr Blood Cancer 66(6):e27658.

7. Finlay J, da Silva NS, Lavey R, Bouffet E, Kellie SJ, Shaw E, Saran F, Matsutani M (2008) The management of patients with primary central nervous system (CNS) germinoma: current controversies requiring resolution. Pediatr Blood Cancer 51:313-316.

8. Murray MJ, Bartels U, Nishikawa R, Fangusaro J, Matsutani M, Nicholson JC (2015) Consensus on the management of intracranial germ-cell tumours. Lancet Oncol 16(9):e470-e477.

9. Balmaceda C, Heller G, Rosenblum M, Diez B, Villablanca JG, Kellie S, Maher P, Vlamis V, Walker RW, Leibel S, Finlay JL (1996) Chemotherapy without irradiation-a novel approach for newly diagnosed CNS germ cell tumors: results of an international cooperative trial. The first international central nervous system germ cell tumor study. J Clin Oncol 14:2908-2915.

10. ] Kellie SJ, Boyce H, Dunkel IJ, et al. Intensive cisplatin and cyclophosphamide-based chemotherapy without radiotherapy for intracranial germinomas: failure of a primary chemotherapy approach. Pediatr Blood Cancer 2004;43(2): 126e133. 
11. Da Silva NS, Cappellano AM, Diez B, Cavalheiro S, Gardner S, Wisoff J, et al. Primary chemotherapy for intracranial germ cell tumors: results of the third international CNS germ cell tumor study. Pediatr Blood Cancer 2010;54(3):377e383.

12. ] Bamberg M, Kortmann RD, Calaminus G, Becker G, Meisner C, Harms D, et al. Radiation therapy for intracranial germinoma: results of the German cooperative prospective trials MAKEI 83/86/89. J Clin Oncol 1999;17(8):2585e2592.

13. Kim JY, Park J. Understanding the Treatment Strategies of Intracranial Germ Cell Tumors: Focusing on Radiotherapy. J Korean Neurosurg Soc. 2015 May;57(5):315-22.

14. Rogers SJ, Mosleh-Shirazi MA, Saran FH. Radiotherapy of localised intracranial germinoma: time to sever historical ties? Lancet Oncol. 2005 Jul;6(7):509-19.

15. Calaminus G., Bamberg M., Baranzelli M.C., Benoit Y., di Montezemolo L.C., Fossati-Bellani F., Jürgens H., Kühl H.J., Lenard H.G., Lo Curto M., et al. Intracranial germ cell tumors: A comprehensive update of the European data. Neuropediatrics. 1994;25:26-32.

16. Cheng S, Kilday JP, Laperriere N, Janzen L, Drake J, Bouffet E, et al (2016) Outcomes of children with central nervous system germinoma treated with multi-agent chemotherapy followed by reduced radiation. J Neurooncol 127(1):173-80.

17. Takami H, Fukuoka K, Fukushima S, Nakamura T, Mukasa A, Saito N, et al (2019) Integrated clinical, histopathological, and molecular data analysis of 190 central nervous system germ cell tumors from the iGCT Consortium. Neuro Oncol 21(12):1565-1577

18. Calaminus G, Kortmann R, Worch J, Nicholson JC, Alapetite C, Garrè ML, et al (2013) SIOP CNS GCT 96: final report of outcome of a prospective, multinational nonrandomized trial for children and adults with intracranial germinoma, comparing craniospinal irradiation alone with chemotherapy followed by focal primary site irradiation for patients with localized disease. Neuro Oncol 15(6):788-96.

19. Calaminus G, Frappaz D, Kortmann RD, Krefeld B, Saran F, Pietsch T, et al (2017) Outcome of patients with intracranial non-germinomatous germ cell tumors-lessons from the SIOP-CNS-GCT-96 trial. Neuro Oncol 19(12):1661-1672. 
20. Biswas A, Julka PK, Bakhshi S, Singh M, Rath GK (2017) Treatment Outcome in Patients with Primary Central Nervous System Germ Cell Tumour: Clinical Experience from a Regional Cancer Centre in North India. Pediatric neurosurgery52(4):240-249.

21. Worawongsakul R, Sirachainan N, Rojanawatsirivej A, Boongird A, Singhsnaeh A, Swangsilpa $\mathrm{T}$, et al (2020) Carboplatin-based regimen in pediatric intracranial germ-cell tumors (IC-GCTs): effectiveness and ototoxicity. Neurooncol Pract 7(2):202-210.

22. de Rezende ACP, Weltman E, Chen MJ, Helito JK, de Carvalho ÍT, Sakuraba RK, et al (2019) Intensity-modulated ventricular irradiation for intracranial germ-cell tumors: Survival analysis and impact of salvage re-irradiation. PLoS One 14(12): e0226350.

23. McMahon SJ (2018) The linear quadratic model: usage, interpretation and challenges. Phys Med Biol 19;64(1):01TR01.

24. Van Leeuwen CM, Oei AL, Crezee J, Bel A, Franken NAP, Stalpers LJA, Kok HP (2018) The alfa and beta of tumours: a review of parameters of the linear-quadratic model, derived from clinical radiotherapy studies. Radiat Oncol 16;13(1):96.

25. Allen JC, Kim JH, Packer RJ (1987) Neoadjuvant chemotherapy for newly diagnosed germ-cell tumors of the central nervous system. J Neurosurg67(1):65-70.

26. Calaminus G, Bamberg M, Baranzelli MC, Benoit Y, di Montezemolo LC, Fossati-Bellani F, et al (1994) Intracranial germ cell tumors: a comprehensive update of the European data. Neuropediatrics25(1):26-32.

27. Douglas JG, Rockhill JK, Olson JM, Ellenbogen RG, Geyer JR. Cisplatin-based chemotherapy followed by focal, reduced-dose irradiation for pediatric primary central nervous system germinomas. J Pediatr Hematol Oncol. 2006;28:36-39. 23 
28. Alapetite C, Brisse H, Patte C, et al. Pattern of relapse and outcome of non-metastatic germinoma patients treated with chemotherapy and limited field radiation: the SFOP experience. Neuro Oncol. 2010; 12:1318-1325.

29. Khatua S, Dhall G, O’Neil S, et al. Treatment of primary CNS germinomatous germ cell tumors with chemotherapy prior to reduced dose whole ventricular and local boost irradiation. Pediatr Blood Cancer. 2010;55: 42-46.

30. Fangusaro J, Wu S, MacDonald S, Murphy E, Shaw D, Bartels U, et al (2019) Phase II Trial of Response-Based Radiation Therapy for Patients with Localized CNS Nongerminomatous Germ Cell Tumors: A Children's Oncology Group Study. J Clin Oncol 37(34):3283-90.

31. Nichols C R, Breeden ES, Loehrer PJ, Williams SD, Einhorn LH (1993) Secondary leukemia associated with a conventional dose of etoposide: review of serial germ cell tumor protocols. J Natl Cancer Inst85(1):36-40.

32.Kollmannsberger C, Beyer J, Droz JP, Harstrick A, Hartmann JT, Biron P, Fléchon A, Schöffski P, Kuczyk M, Schmoll HJ, Kanz L, Bokemeyer C (1998) Secondary leukemia following high cumulative doses of etoposide in patients treated for advanced germ cell tumors. J Clin Oncol 16(10):3386-91.

33. Seah T, Zhang Ch, Halbert J, Prabha S, Gupta S. (2018) The magnitude and predictors of therapy abandonment in pediatric central nervous system tumors in low- and middle-income countries: Systematic review andmeta-analysis. Pediatr Blood Cancer. 2019;66:e27692.

Figure Legends 


\section{FIGURE 1}

Outcome of iGCT (germinoma and NGGCT).

(A) Kaplan-Meier estimates of PFS and OS for germinoma and (B) NGGCT.

(C) Kaplan-Meier estimates of PFS and OS for localized germinoma treated with multiagent chemotherapy followed by reduced-volume radiation field (including FRT and FRT plus WVRT subgroup).

(D) Kaplan-Meier estimates of PFS and (E) OS for localized germinoma treated with multiagent chemotherapy followed by reduced-volume radiation field (including FRT and FRT plus WVRT) between 2000-2009 and 2010-2019.

(F) Kaplan-Meier estimates of PFS for localized germinoma treated with multiagent chemotherapy followed by FRT and localized germinoma treated with multiagent chemotherapy followed by FRT plus WVRT.

P-values are determined using the log-rank method. 


\section{Figures}
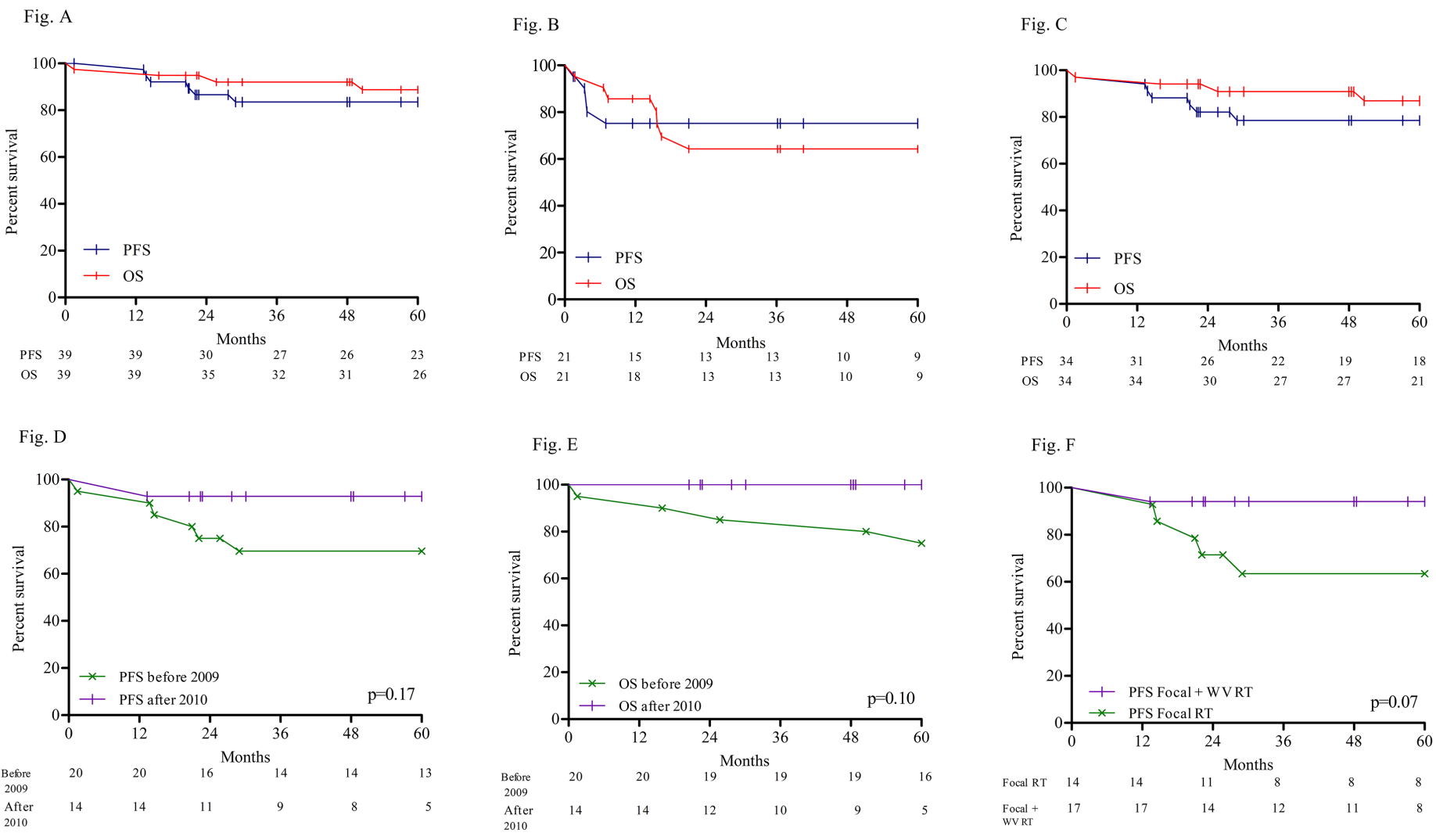

\section{Figure 1}

Outcome of iGCT (germinoma and NGGCT). (A) Kaplan-Meier estimates of PFS and OS for germinoma and (B) NGGCT. (C) Kaplan-Meier estimates of PFS and OS for localized germinoma treated with multiagent chemotherapy followed by reduced-volume radiation field (including FRT and FRT plus WVRT subgroup). (D) Kaplan-Meier estimates of PFS and (E) OS for localized germinoma treated with multiagent chemotherapy followed by reduced-volume radiation field (including FRT and FRT plus WVRT) between 2000-2009 and 2010-2019. (F) Kaplan-Meier estimates of PFS for localized germinoma treated with multiagent chemotherapy followed by FRT and localized germinoma treated with multiagent chemotherapy followed by FRT plus WVRT. P-values are determined using the log-rank method.

\section{Supplementary Files}

This is a list of supplementary files associated with this preprint. Click to download.

- TABLE1Supplementarydata.pdf

- TABLE1.pdf

- TABLE2.pdf

- TABLE3.pdf 
- TABLE4.pdf 